\title{
The use of an oral contraceptive containing ethinylestradiol and drospirenone in an extended regimen over 126 days
}

\author{
Jean-Michel Foidart ${ }^{\mathrm{a}}$, Patricia J. Sulak ${ }^{\mathrm{b}, \mathrm{c}}$, Ilka Schellschmidt ${ }^{\mathrm{d}}$, Doris Zimmermann ${ }^{\mathrm{d}}$ The Yasmin $^{\circledR}$ Extended $^{\circledR}$ \\ Regimen Study Group ${ }^{1}$ \\ ${ }^{a}$ Department of Obstetrics and Gynecology, University of Liège, B-400 Liège, Belgium \\ ${ }^{b}$ Department of Obstetrics and Gynecology, Scott \& White Clinic/Memorial Hospital, Temple, TX 76508, USA \\ ${ }^{c}$ Texas A\&M University System Health Science Center, College of Medicine, Temple, TX 76508, USA \\ ${ }^{d}$ Schering AG, Global Medical Development, 13342 Berlin, Germany
}

\begin{abstract}
Objective: To assess the bleeding profile, acceptance and safety of an extended 126-day regimen of the oral contraceptive Yasmin ${ }^{\circledR}[30 \mu \mathrm{g}$ ethinylestradiol (EE) and $3 \mathrm{mg}$ drospirenone (DRSP)].

Methods: Using daily diaries, 177 women recorded bleeding events throughout the 126-day cycle. At end of treatment, the women completed questionnaires reflecting their satisfaction with the extended regimen. A subset of 30 women underwent endometrial histology sampling after completion of the extended regimen.

Results: Of 177 women assigned to the extended regimen, 80.8\% completed the extended 126-day regimen. Approximately $40 \%$ of the women reported complete absence of bleeding, while in $60 \%$ a shift towards less intense bleeding was observed. The first onset of bleeding occurred after a median of 99.0 days into the extended cycle. The acceptance of the extended regimen was high, with $68.4 \%$ of the women expressing satisfaction. The general safety profile with the extended use was comparable to that seen with the conventional 21+7-day regimen. All endometrial biopsies with sufficient material for analysis were normal and supported the endometrial safety of the extended regimen.

Conclusion: This study showed that the continuous use of a 30- $\mu \mathrm{g}$ EE and 3-mg DRSP formulation over 126 days was safe, efficacious, well
\end{abstract}

Keywords: Extended cycle; Oral contraceptive; Breakthrough bleeding; Endometrial safety; Drospirenone

\section{INTRODUCTION}

To date, most combination oral contraceptives (COCs) include a dosage regimen that induces withdrawal bleeding every 4 weeks. This concept was first introduced by Dr. Gregory Pincus in the late 1950s in order to mimic the natural "lunar" cycle that usually lasts between 25 and 30 days [1]. The administration of COCs in a 21-day cycle of active hormone pills followed by a 7-day pill-free interval produces predictable withdrawal bleeding in most users.

However, there is no scientific rationale for the 21+7-day cycle as the only regimen for COCs [2]. Prolonged intervals between withdrawal bleedings are established with long-acting contraceptive injectables, implants or progestogen-containing intrauterine devices. In fact, almost 30 years ago it was shown that the pill too has the ability to control the pattern of uterine bleeding beyond 3 weeks without compromising its contraceptive efficacy [3].

In actual clinical practice, the continuous administration of $\mathrm{COC}$ is often recommended to women to postpone withdrawal bleeding for medical reasons such as dysmenorrhea, excessive blood loss or hormone withdrawal symptoms, and for personal reasons such as holidays or sports [4]. Meanwhile, numerous studies on this issue have been performed proving that an extended regimen is initially associated with an increased rate of irregular bleeding, while the total number of bleeding days can be reduced significantly [5-8]. Reports of extended cycle

\footnotetext{
${ }^{1}$ Yasmin ${ }^{\circledR}$ Extended Regimen Study Group: Lars-Olof Abrahamson, MD, Hvidovre, Denmark; Marc Dhont, MD, Gent, Belgium; Christine Felding, MD, Allerod, Denmark; Elisabeth Ingelhammar, MD, Göteborg, Sweden; Tore Lunde, MD, Larvik, Norway; Mette Haase Moen, MD, Trondheim, Norway, Sven-Eric Olsson, MD, Danderyd, Sweden; Eduard Pauwels, MD, Brasschaat, Belgium, Hanne Rem, MD, VærlØse, Denmark Yasmin ${ }^{\circledR}$ is a registered Trademark of Schering AG, Berlin, Germany
} 
regimens in an actual clinical practice have confirmed the acceptance of delaying the onset of withdrawal bleeding and associated hormone withdrawal symptoms $[9,10]$. Recent surveys revealed that women indeed accept this concept. When queried, $65 \%$ of Dutch women between 25 and 34 years preferred bleeding every 3 months or less often, and 31\% preferred not to bleed [11]. Although extended COC regimens are commonly recommended by physicians, only in the USA is OC available that is specifically labeled for 84 days of extended use $[5-8,12]$.

We initiated this study to investigate the bleeding pattern, efficacy and safety of a 126-day extended regimen, and to test whether extension beyond 3 months is a favorable treatment option for COC users. The 126-day extended regimen consists of a known combination of $30 \mu \mathrm{g}$ ethinylestradiol (EE) and $3 \mathrm{mg}$ drospirenone (DRSP) (Yasmin ${ }^{\circledR}$, Schering) at a dose level of proven efficacy and safety $[13,14]$. Because this formulation has been shown to reduce water retention, weight gain, premenstrual symptoms and skin disorders such as acne when used in a 21+7-day fashion, it was chosen as a possible ideal candidate for an extended cycle regimen [1517].

\section{MATERIAL AND METHODS}

\subsection{Study design and population}

This was a prospective, multicenter, open, uncontrolled study of the 126-day extended regimen of the oral contraceptive Yasmin ${ }^{\circledR}(30 \mu \mathrm{g}$ EE and $3 \mathrm{mg}$ DRSP). The study was conducted from May 2002 to January 2004 at 10 study centers in Belgium, Denmark, Sweden and Norway, consisting of private practices and universityaffiliated clinics. The study protocol was approved by appropriate ethics committees of the participating study sites and was conducted in accordance with the ethical principles that have their origin in the Declaration of Helsinki and the ICH-GCP Guidelines of 17 January 1997.

Women were excluded for age less than 18 years or greater than 35 years (maximum age for smokers was 30 years), current OC use of less than six cycles and standard contraindications to COC use. After the written informed consent was obtained, information on demographics, medical and gynecological history, OC use history and concomitant medication was collected. Height, weight and blood pressure were obtained along with a physical and gynecological examination, including a Papanicolaou smear if no normal result in the last 3 months was available.

\subsection{Treatment and procedures}

In order to prevent carryover effects from any preceding COC, the women switched to two 21+7-day run-in cycles of the EE/DRSP formulation before beginning the extended cycle. After completion of the run-in cycles, the women were advised to continue daily tablet intake without any tablet-free intervals for 126 days. The women were to record the daily pill intake and vaginal bleeding in their diary cards.

Women returned for clinic visits every 6 weeks and at study exit, except for one phone visit after 6 weeks of continuous use. They were instructed to bring their diary cards and empty pill packs to each visit. Investigators verified the diary records and signed for completeness and plausibility. Vital signs, adverse events and changes in concomitant medication were assessed at each visit. A thorough physical and gynecological examination including a Papanicolaou smear was performed at study exit. At end of treatment, the women completed a questionnaire to assess their satisfaction with the treatment using a seven-point scale ranging from "very much," "much," "minimally," "neither/nor," "satisfied" to "minimally," "much" and "very much dissatisfied." They were also asked which treatment option they would prefer for future use (extended regimen, conventional 21+7-day cycle or other options including other regimens or other methods of contraception).

After completion of the 126-day cycle, women could either exit the study or continue with the extended regimen for another 126-day cycle without any break. One participating study center was randomly selected to perform endometrial biopsies on women who had completed the 252-day regimen and volunteered to sampling. A single pathologist scored the specimen for adequacy and histologic findings. The results were classified as insufficient material, normal/nonsuspicious or abnormal.

\subsection{Efficacy assessments and analysis}

Efficacy measures were primarily based on bleeding patterns such as time to first bleeding day (requiring sanitary protection), number of bleeding or spotting days, and the maximum bleeding intensity. The bleeding 
intensity for any given day was scored (none, spotting=no sanitary protection needed, light=less than associated with normal menstruation with need for sanitary protection, normal=like normal menstruation, heavy=more than normal menstruation). For each woman, the bleeding patterns were analyzed for the entire 126-day cycle, and for the World Health Organization (WHO)-recommended reference period of 90 days; assessing the first 90 days of the 126-day interval [18]. In addition, the number of women with missed pills, the treatment acceptance and the preferred regimen options for future use were analyzed. Safety measures included adverse event reports and general safety assessments such as gynecological examinations, cervical smears and endometrial biopsies. All collected data were computerized and stored in an Oracle database. The data organization and the statistical evaluation of the clinical trial were performed using the statistical software package SAS 8.2 on a Windows platform. All variables were analyzed by adequate descriptive statistics, i.e., number of observations, mean, standard deviation, minimum, median, maximum for continuous data and frequency tables for categorical data. No hypotheses have been stated.

\section{RESULTS}

\subsection{Study population and disposition}

A total of 184 women - all of them current OC users - were recruited and treated across 10 study centers. The enrollment demographics and baseline characteristics of the study population are displayed in Table 1 . The great majority of women were Caucasian $(98.4 \%)$ and non-smokers $(76.1 \%)$. They were $23.7 \pm 4.1$ years old (mean \pm $\mathrm{SD})$. At time of enrollment, the women rated their monthly withdrawal bleeding as predominantly normal in intensity. The duration of the monthly bleeding varied from 2 to 8 days ( $4.7 \pm 1.0$ days).

Three of 184 women taking study medication were lost to follow-up. Of the remaining 181,175 were assigned to run-in cycles, while 6 omitted the run-in as they were already taking the study medication for at least two cycles. An overview of study completion rates and reasons for dropout is given in Fig. 1. Of 177 women admitted to the 126-day cycle, $143(80.8 \%)$ completed the extended regimen. The most common reason for premature discontinuation was adverse events, most frequently specified as bleeding. With completion of the 126-day cycle, women were given the option to either stop or continue the extended regimen for another 126 days.

All 177 women, who received at least one dose of the 126 - day regimen and for whom diary information was available, were included in the analysis of the compliance, bleeding pattern, acceptance of extended regimen and safety.

Table 1- Enrollment demographic information (full analysis set)

\begin{tabular}{ll}
\hline Characteristics & Extended cycle $(N=184)$ \\
\hline Race & $181(98.4)$ \\
Caucasian, $n(\%)$ & $1(0.5)$ \\
Hispanic, $n(\%)$ & $2(1.1)$ \\
Asian, $n(\%)$ & $23.7(4.1)$ \\
Mean age, years (SD) & $22.0(3.1) ; 15.8-31.4$ \\
Mean BMI $(\mathrm{SD}) ;$ range $\left(\mathrm{kg} / \mathrm{m}^{2}\right)$ & $44(23.9)$ \\
Current smokers, $n(\%)$ & \\
Withdrawal bleeding & $4.7(1.0) ; 2-8$ \\
$\quad$ Mean lengths (SD); range (days) & $45(24.5)$ \\
Light intensity ${ }^{\mathrm{b}}, n(\%)$ & $128(69.6)$ \\
Normal intensity ${ }^{\mathrm{b}}, n(\%)$ & $11(6.0)$ \\
$\quad$ Heavy intensity ${ }^{\mathrm{b}}, n(\%)$ & \\
${ }^{\mathrm{a}}$ BMI=Body mass index. & \\
${ }^{\mathrm{b}}$ By maximum intensity. &
\end{tabular}


Fig. 1. Study population flow chart and reasons for discontinuation.

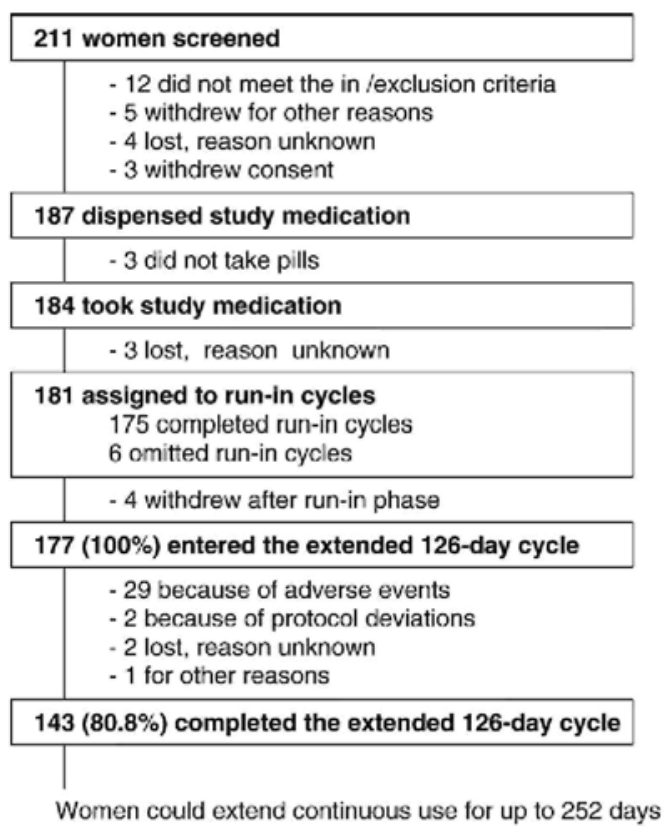

\subsection{Compliance and contraceptive reliability}

Based on the women's diary study medication records, pill compliance within the 126-day regimen was measured as the number of women missing more than two tablets continuously, or as the number of women missing more than four tablets noncontinuously. A total of 19 women (10.7\%) were deemed noncompliant. No pregnancy was reported during the entire course of the study.

\subsection{Bleeding pattern}

All bleeding information, from run-in phase throughout the extended 126-day regimen, was derived from the women's daily diary entries using established criteria for different bleeding patterns. Accordingly, Fig. 2 presents the percentage of women who reported bleeding by cycle day. During the run-in phase, two withdrawal bleeding episodes were expected to occur, while during the extended regimen any bleeding or spotting was considered unscheduled (breakthrough) bleeding/spotting. The per-day analysis indicated that the percentage of women reporting unscheduled bleeding increased slightly within the first 50 days, but then seemed to plateau in the further course of the extended regimen. Furthermore, the per-day analysis revealed that more than half of the women had started the extended regimen while the scheduled withdrawal bleeding from run-in persisted. As a result of this, there was an overlap of withdrawal bleeding of the second run-in cycle into the first 2 weeks of the extended regimen. For the analysis of the first onset of breakthrough bleeding and its intensity, it was decided to disregard these 2 weeks of the extended regimen.

Table 2 summarizes the varying degrees of reported breakthrough bleeding. With continuous use over 126 days, bleeding was completely prevented in almost $40 \%$ of the women, while $60 \%$ reported at least 1 unscheduled bleeding day. However, typically, the bleeding was predominately light in intensity (31.6\% had maximum light bleeding, 24.3\% normal bleeding and 5.6\% heavy bleeding). The first onset of bleeding occurred after a median of 99.0 days into the extended regimen.

Summary statistics (median, first and third quartiles) for the diary-reported number of bleeding and/or spotting days are presented in Table 3. Here, the scheduled withdrawal bleeding that occurred within the first 14 days of the extended regimen was included in the analysis, in order to account for the total degree of bleeding per indicated time interval, irrespective of whether this was attributed to scheduled or unscheduled bleeding. Out of 126 possible days, the median total number of days with bleeding was 3.0 days vs. median 9.0 days with spotting only. 
Fig. 2. Percentage of all women reporting bleeding by study day.

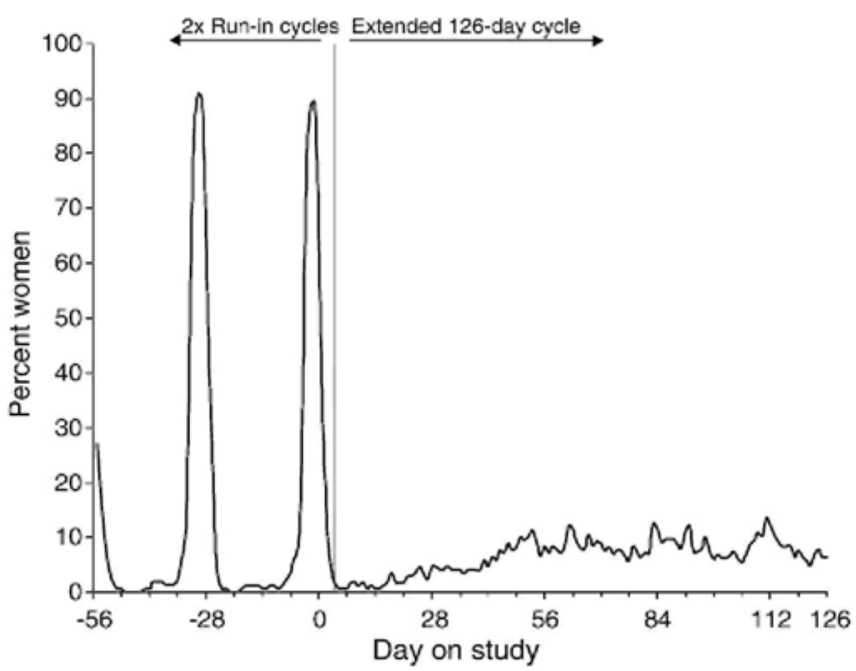

For the comparison of bleeding patterns with various forms of $\mathrm{OC}$, the WHO recommends the use of fixed reference periods for quantification of vaginal bleeding [18]. Therefore, the bleeding data from the menstrual diaries were also analyzed using a 90-day reference period approach. No major difference was revealed when comparing the bleeding pattern reported during the 90-day or the 126-day interval. The number of bleeding-only days was essentially unchanged, with median 2.0 for the 90-day interval and 3.0 days for the entire 126-day regimen. Likewise, with continuous use over 90 or 126 days, the total number of bleeding and/or spotting days was also proportional to the number of days evaluated (median 13.0 vs. 17.0 days). The number of sporting-only days, however, seems to increase to some extent. With approximately one-and-a half times the number of treatment days (90 vs. 126 days), the number of sporting-only days doubles (median 4.0 vs. 9.0 days). In accordance with recommendations by the Food and Drug Administration, the bleeding/spotting pattern was further investigated by calculating the number of women with $\geq 7$ and $\geq 20$ days of bleeding/spotting [8]. Seven days or more of bleeding/spotting were observed by $55.9 \%$ of the women during 90 days and by $72 \%$ during 126 days; 20 days or more by $27.1 \%$ and $45.2 \%$, respectively.

Taken together, the bleeding pattern observed during the 90-day and the 126-day interval suggests that breakthrough bleeding/spotting increased over time only slightly, and, typically, this shift was attributed to spotting only.

\subsection{Treatment acceptance}

At study exit, the women were queried about their satisfaction with the extended regimen of the EE/DRSP formulation (Fig. 3). The acceptance of this extended regimen was high, with $68.4 \%$ of all users giving a positive rating ("very much satisfied" given by $19.2 \%$, "much" by $35.6 \%$ and "minimally" by $13.6 \%$ ). Dissatisfaction was expressed by $21.9 \%$ of the women (with ratings of "very much dissatisfied" by $3.3 \%$, "much" by $12.4 \%$ or "minimally" by $6.2 \%)$.

When asked what type of birth control they planned to use in the future, $42.4 \%$ preferred to continue with an extended regimen, while $48.6 \%$ wanted to change back to a conventional $21+7$-day cycle regimen, and $5.6 \%$ chose other options. Other options included no contraception because of wish for pregnancy $(2.3 \%)$ or intrauterine devices $(2.8 \%)$. Approximately one third of all women in the study, and the great majority of the women who planned to continue with an extended regimen, preferred the study regimen of 126 days, while approximately $10 \%$ wished to continue with an extended regimen, however, of shorter duration, e.g., 3 or 2 months, or wanted to alternate conventional $21+7$-day cycles with periods of continuous pill-intake.

\subsection{Safety}

Generally, the adverse event profile with the continuous use of the EE/DRSP formulation was similar to that seen with the conventional 21+7-day regimen. In this study, adverse events most frequently reported were symptoms associated with sinus and respiratory tract infections (typically reported as sinusitis, pharyngitis or flu syndrome), irregular bleeding, breast pain and headache. As expected with an extended regimen, irregular bleeding occurred; however, the majority of these events were rated as mild in intensity [mild in $22(12.4 \%)$ 
women, moderate in $10(5.6 \%)$ ] and no severe bleeding. In 19 (10.7\%) of these cases, irregular bleeding led to premature discontinuation of study medication. A 36-year-old nonsmoking woman, who had been using OCs for 10 years, experienced cholelithiasis requiring surgery approximately 9 months after the start of the extended 126day regimen. She completed study medication, followed by further COC treatment with the same marketed EE/DRSP formulation.

No appreciable changes in vital signs were observed over the course of the study, nor was there any finding that gave cause for concern in the physical, breast or gynecological examinations at screening, end of treatment or withdrawal.

In order to assess the effect of the extended administration of EE/DRSP on the endometrium, a subgroup of 30 women at one center underwent endometrial histology sampling at completion of treatment. Only women who had chosen to prolong the extended regimen to a total of 252 days were selected. In eight cases, the tissue obtained was insufficient for diagnosis. All other endometrial biopsies were in accordance with the typical endometrial changes caused by a COC (in 22 of 30 women with sufficient tissue for diagnosis) and supported the endometrial safety of continuous use of the EE/DRSP formulation for up to 252 days.

Table 2- Diary reports of bleeding profile using established criteria for bleeding intensities (full analysis set)

\begin{tabular}{|c|c|}
\hline Women reporting bleeding & Extended cycle continuous use over 126 days $(N=177)$ \\
\hline At least 1 bleeding day, $n(\%)$ & $109(61.6)$ \\
\hline Light intensity $^{\mathrm{a}}, n(\%)$ & $56(31.6)$ \\
\hline Normal intensity ${ }^{\mathrm{a}}, n(\%)$ & $43(24.3)$ \\
\hline Heavy intensity ${ }^{\mathrm{a}}, n(\%)$ & $10(5.6)$ \\
\hline Time to first bleeding ${ }^{\mathrm{b}}$, median days ( $\left.\mathrm{q} 25, \mathrm{q} 75\right)$ & $99.0(51.0,127.0)$ \\
\hline
\end{tabular}

Table 3- Diary reports of observed breakthrough bleeding and spotting days for all women (full analysis set; $N=$ 177)

\begin{tabular}{lll}
\cline { 2 - 3 } & \multicolumn{2}{c}{ Extended cycle continuous use over } \\
\cline { 2 - 3 } & 126 days $(N=177)$ & 90 days $^{\mathrm{a}}(N=177)$ \\
\hline Bleeding days, median $(\mathrm{q} 25, \mathrm{q} 75)$ & $3.0(1.0,10.0)$ & $2.0(0.0,7.0)$ \\
Spotting days, median $(\mathrm{q} 25, \mathrm{q} 75)$ & $9.0(2.0,21.0)$ & $4.0(2.0,7.0)$ \\
Bleeding/spotting days, median $(\mathrm{q} 25, \mathrm{q} 75)$ & $17.0(5.0,32.0)$ & $13.0(10.0,18.0)$ \\
Women with $\geq 7$ days, $n(\%)$ & $127(71.7)$ & $99(55.9)$ \\
Women with $\geq 20$ days, $n(\%)$ & $80(45.2)$ & $48(27.1)$ \\
\hline${ }^{\mathrm{a}}$ In accordance with the WHO-recommended reference period of 90 days; assessing the first 90 days of the 126-day interval.
\end{tabular}

\section{DISCUSSION/CONCLUSION}

This large-scale, prospective study showed that the extension of the conventional 21+7-day OC cycle with a 30$\mu \mathrm{g}$ EE and 3-mg DRSP formulation to 126 days was safe, well accepted by the majority of users and resulted in considerably fewer bleeding days. With continuous use over 126 days, bleeding was completely suppressed in approximately $40 \%$ of the 177 women. In the $60 \%$ reporting breakthrough bleeding, a shift towards less intense bleeding was achieved. While the majority of women rated their monthly withdrawal bleeding as normal in intensity on the 21+7-day regimen, bleeding with the 126-day regimen was predominately light in intensity. The first onset of bleeding occurred after a median of 99.0 days into the extended cycle.

Since 1977, when the first study on the efficacy and acceptance of long-cycle COC regimen had been carried out, numerous studies with different EE/progestogen combinations proved that bleeding can be reduced significantly while unscheduled breakthrough bleeding often increases, typically, during the first months of treatment $[3,6,7,19]$. Therefore, physicians counseling women on extended regimens and the users need to balance the convenience of fewer scheduled withdrawal bleeding against the inconvenience of slightly more 
breakthrough bleeding or spotting with an extended regimen — knowing that the individual susceptibility to breakthrough bleeding or spotting is difficult to predict. For that reason, it is important for physicians to guide women on how to take and what to expect when considering the option of an extended regimen. The present study confirms the ability and acceptance of COC users to switch their usual 21+7-day cycles to an extended 126-day regimen with DRSP and may help health care providers to adequately counsel their patients seeking to reduce the number of monthly bleedings.

Fig. 3. Treatment acceptance and preferred options for future use.
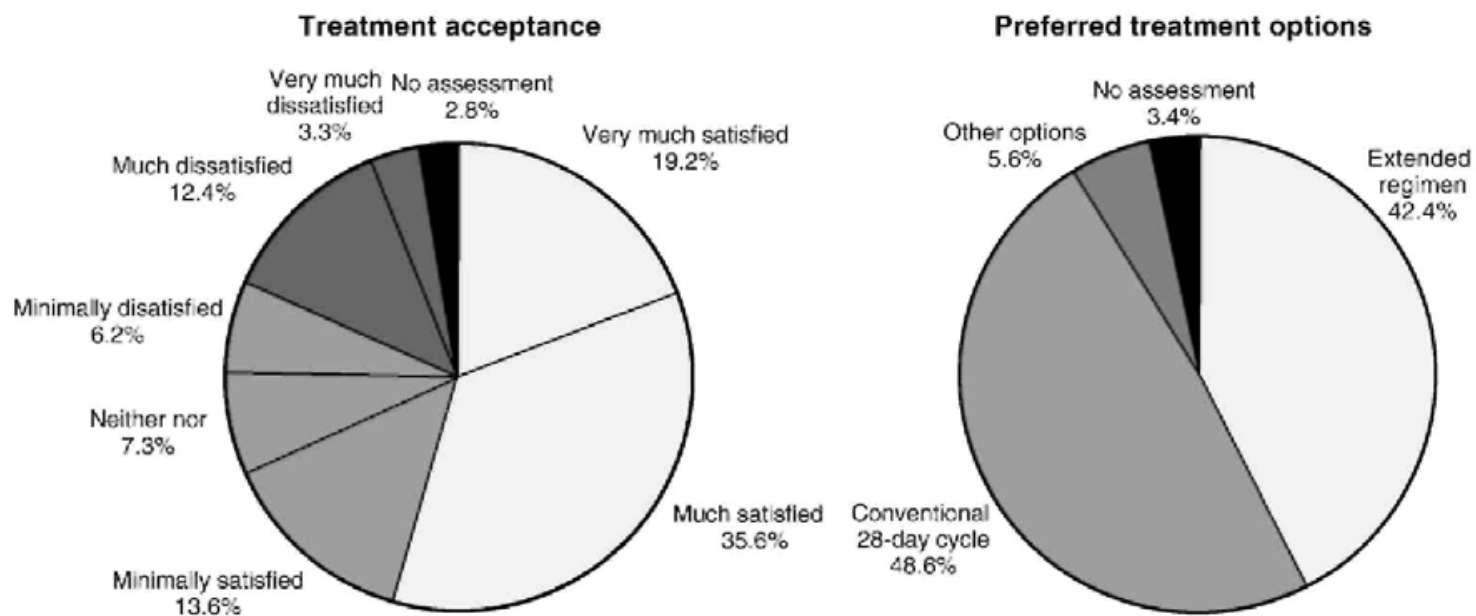

In this study, the women switched to the 126-day extended regimen after two 21+7-day run-in cycles of the same EE/DRSP formulation. Although it cannot be ruled out that the bleeding profile may have been positively affected, as no COC "starters" were included in the study (who are generally at a higher risk of unscheduled bleeding at the beginning), we do believe that this has no essential impact on the overall interpretation of study results. In contrast, the population in this study reflects both the population of other studies investigating the extended COC use $[5,7,9,10,19]$ and real life of contraceptive counseling where most providers discuss the possibility of this regimen with COC users and most women considering an extended regimen have some experience with OCs.

Our study clearly demonstrated that an OC containing EE/DRSP in an extended 126-day regimen is a suitable option for many women. Besides individual variation, which is commonly observed with any OC use, the extended 126-day regimen reduced the degree of bleeding/ spotting considerably. With continuous use of this EE/DRSP formulation, the women noted median 13.0 bleeding/ spotting days compared with median 18.0 days using the same COC in a conventional 21+7-day regimen (based on a 90-day reference period, data on file).

In 2003, Anderson and Hait [5] reported study results of an 84+7-day COC containing $30 \mu \mathrm{g}$ EE and $150 \mu$ levonorgestrel (Seasonale ${ }^{\circledR}$, Barr Labs, Pamona, NY, USA). This study revealed that breakthrough bleeding and/ or spotting decreased with every subsequent $84+7$ cycles, being greatest during the first two cycles. With Seasonale ${ }^{\circledR}$, women reported median 12.0 days of breakthrough bleeding/spotting during the first 84-day cycle. With continuous use of the EE/DRSP formulation over 90 days, the median number of bleeding/spotting was similar (median 13.0 vs. 12.0 days); however, the percentage of women with $\geq 7$ or $\geq 20$ days of bleeding/spotting seems lower with EE/DRSP. During the first 84 days on Seasonale ${ }^{\circledR}, 65 \%$ of the women experienced $\geq 7$ days of bleeding/spotting vs. $55.9 \%$ of the women using the EE/DRSP formulation (Yasmin ${ }^{\circledR}$ ) continuously over 90 days [8]. Twenty days or more were reported by $35 \%$ of the women taking Seasonale $\AA$ and by $27.1 \%$ taking Yasmin ${ }^{\circledR}$ continuously. When compared with a 336-day extended regimen of a $20-\mu \mathrm{g} E E$ and $100-\mu \mathrm{g}$ levonorgestrel formulation, the reported number of bleeding and spotting days from Day 1 to Day 84 was similar to that from Day 1 to Day 126 in this study (with median 3.0 and 9.0 days, respectively) [7].

Moreover, the comparison between the 90-day and the 126-day interval of the extended regimen with the EE/DRSP formulation indicates that in the majority of women the overall degree of breakthrough bleeding/spotting was essentially unchanged throughout the extended 126-day cycle. Since breakthrough 
bleeding/spotting usually diminishes with consecutive COC cycles-standard 21+7 as well as extended regimens - we recommend multiple extended 126-day regimens of EE/DRSP with an intervening hormone-free interval as a favorable treatment option. Consistently, the great majority of the women in this study who planned to continue with an extended regimen, preferred the study regimen, while only $10 \%$ wished to continue with an extended regimen of shorter duration.

The EE/DRSP formulation used in this study is proven to be an effective and safe method of fertility control with cyclic OC use $[13,14]$. An extended COC regimen should provide better contraceptive reliability in the event of missed tablets due to the greater potential for sustained ovarian suppression [20]. When taken daily over 126 days, pregnancy was prevented in all 177 women including those 19 women who missed more than two tablets continuously or more than four noncontinuously.

The adverse event profile of the extended 126-day regimen was similar to that of other extended regimens and generally comparable to those of other COCs $[5,13,14]$. As expected with an extended regimen, higher rates of bleeding-reported events were observed compared to conventional regimens, emphasizing the need to reinforce this issue during counseling. The adverse event profile was also favorable in those women who had completed the extended 252-day regimen, although the number of women reporting complete absence of bleeding was reduced considerably. Endometrial histology was investigated in a small subgroup of our users. In all 22 women where sufficient material was available, findings were in accordance with the typical endometrial changes caused by a COC, providing reassurance that regular withdrawal bleedings are not necessary to prevent endometrial hyperplasia [21]. Similar results were found with the 336-day extended regimen of a $20-\mu \mathrm{g}$ EE and $100-\mu \mathrm{g}$ levonorgestrel formulation in which endometrial biopsies were carried out in eight women revealing inactive or atrophic samples in seven women [7].

In summary, the continuous use of a 30- $\mu \mathrm{g}$ EE and 3-mg DRSP formulation over 126 days was efficacious, well tolerated and resulted in considerable reduction of bleeding days. Although limited by numbers and duration of use, the present study confirms the extended 126-day regimen as a favorable treatment option in many women who desire to reduce withdrawal bleedings markedly.

\section{Acknowledgment}

We wish to thank Dietmar Trummer and Klaas Heine-mann (Schering AG) for statistical assistance in the preparation of this manuscript. We are also indebted to E. Pauwels (Belgium) for endometrial histology assessments.

\section{References}

[1] Pincus G, Garcia CR, Rock J, et al. Effectiveness of an oral contraceptive; effects of a progestin-estrogen combination upon fertility, menstrual phenomena, and health. Science 1959;130:81-3

[2] Thomas SL, Ellertson C. Nuisance or natural and healthy: should monthly menstruation be optional for women? Lancet 2000;355: 922-4.

[3] Loudon NB, Foxwell M, Potts DM, Guild AL, Short RV. Acceptability of an oral contraceptive that reduces the frequency of menstruation: the tri-cycle pill regimen. BMJ 1977;2:487-90.

[4] Shakespeare J, Neve E, Hodder K. Is norethisterone a lifestyle drug? Results of database analysis. BMJ 2000;320:291.

[5] Anderson FD, Hait H. A multicenter, randomized study of an extended cycle oral contraceptive. Contraception 2003;68:89-96.

[6] Kwiecien M, Edelman A, Nichols MD, Jensen JT. Bleeding patterns and patient acceptability of standard or continuous dosing regimens of a low-dose oral contraceptive: a randomized trial. Contraception 2003;67:9-13.

[7] Miller L, Hughes JR Continuous combination oral contraceptive pills to eliminate withdrawal bleeding: a randomized trial. Obstet Gynecol 2003;101:653-61.

[8] U.S. Food and Drug Administration. Drug approval package for Seasonale (levonorgestrel/ethinyl estradiol) tablets. (created 03/24/ 2004) Internet Commun 2004 http://www.fda.gov/cder/foi/nda/2003/ 21 -544_Seasonale.htm.

[9] Sulak PJ, Kuehl TJ, Ortiz M, Shull BL. Acceptance of altering the standard 21-day/7-day oral contraceptive regimen to delay menses and reduce hormone withdrawal symptoms. Am J Obstet Gynecol 2002;186:1142-9. 
Published: Contraception (2006), vol.73, iss.1, pp. 34-40

Status: Postprint (Author's version)

[10] Sulak PJ, Carl J, Gopalakrishnan I, Coffee A, Kuehl TJ. Outcomes of extended oral contraceptive regimens with a shortened hormonefree interval to manage breakthrough bleeding. Contraception 2004;70: 281-7.

[11] den Tonkelaar I, Oddens BJ. Preferred frequency and characteristics of menstrual bleeding in relation to reproductive status, oral contraceptive use, and hormone replacement therapy use. Contraception 1999; 59:357-62.

[12] U.S. Food and Drug Administration. Birth control guide. (Updated December 2003) FDA Consum 2004 http://www.fda.gov/fdac/ features/1997/babytabl.html.

[13] Foidart JM, Wuttke W, Bouw GM, Gerlinger C, Heithecker R. A comparative investigation of contraceptive reliability, cycle control and tolerance of two monophasic oral contraceptives containing either drospirenone or desogestrel. Eur J Contracept Reprod Health Care $2000 ; 5: 124-34$

[14] Huber J, Foidart JM, Wuttke W, et al. Efficacy and tolerability of a monophasic oral contraceptive containing ethinylestradiol and drospirenone. Eur J Contracept Reprod Health Care 2000;5: 25-34.

[15] Parsey KS, Pong A. An open-label, multicenter study to evaluate Yasmin, a low-dose combination oral contraceptive containing drospirenone, a new progestogen. Contraception 2000;61:105-11.

[16] Thorneycroft H, Gollnick H, Schellschmidt I. Superiority of a combined contraceptive containing drospirenone to a triphasic preparation containing norgestimate in acne treatment. Cutis 2004; 74:123-30.

[17] van Vloten WA, van Haselen CW, van Zuuren EJ, Gerlinger C, Heithecker R. The effect of 2 combined oral contraceptives containing either drospirenone or cyproterone acetate on acne and seborrhea. Cutis 2002;69:2-15.

[18] Belsey EM, Machin D, d'Arcangues C. The analysis of vaginal bleeding patterns induced by fertility regulating methods. World Health Organization Special Programme of Research, Development and Research Training in Human Reproduction. Contraception 1986; 34:25360 .

[19] Sulak PJ, Cressman BE, Waldrop E, Holleman S, Kuehl TJ. Extending the duration of active oral contraceptive pills to manage hormone withdrawal symptoms. Obstet Gynecol 1997;89: 179-83.

[20] Coney P, DelConte A. The effects on ovarian activity of a monophasic oral contraceptive with 100 microg levonorgestrel and 20 microg ethinyl estradiol. Am J Obstet Gynecol 1999;181:53-8.

[21] Ludicke F, Johannisson E, Helmerhorst FM, et al. Effect of a combined oral contraceptive containing $3 \mathrm{mg}$ of drospirenone and 30 microg of ethinyl estradiol on the human endometrium. Fertil Steril 2001;76:102-7. 